\title{
THE BIM-BASED INFORMATION INTEGRATION SPHERE FOR CONSTRUCTION PROJECTS
}

\author{
Chung-Wei Feng ${ }^{1}$, Osama Mustaklem ${ }^{1}$, and Yi-Jao Chen ${ }^{2}$ \\ ${ }^{1}$ Department of Civil Engineering, National Cheng Kung University, Tainan, Taiwan \\ ${ }^{2}$ Department of Asset Management and Urban Planning, Kang Ning University, Tainan, Taiwan \\ *Corresponding author (cfeng@mail.ncku.edu.tw)
}

\begin{abstract}
Building Information Modeling (BIM) has been employed in the AEC industry to provide the data repository for the various stakeholders of construction projects in past years. For example architects use it to streamline the information transition throughout the design phases of a project, structural and MEP engineers use BIM as the database for their design projects, and project engineers use it to automatically and dynamically perform quantity takeoffs. Although BIM has respectively contributed to different sectors in the AEC industry, it still hasn't been well exploited to offer an efficient information management solution for the construction phase of a project. This study develops an information integration sphere (IIS) based on the BIM technology to provide a more efficient and effective information integration model. IIS can clearly identify the data requirements of various stakeholders at different stages of the construction project. It also serves as a tool that can better generate, manipulate, and visualize the flow of information along different processes. In addition, IIS can establish the responsibilities of various stakeholders in terms of providing and receiving information within the construction project. With the help of IIS, BIM model can be better built for smoothly transiting and integrating information among different phases of the construction project. An example is presented to show the benefits of applying IIS to managing the information of a project using BIM technologies and laying the path for a smoother transition of data flow between the different stages of the project lifecycle.
\end{abstract}

Keywords: BIM, Information Integration Model

\section{INTRODUCTION}

Although many researchers and organizations have developed guidelines and frameworks for BIM adoption into the AEC industry [1,2], outlining the added value benefit of BIM to the industry as a whole (and not as a desperate set of technologies), and even though these researches have contributed to this field of study, they still lack in developing an information model to effectively map different BIM processes and stakeholders, and integrate the information resulting from the various data exchanges and interaction between them.

In the early development of BIM technology, single project stakeholders have taken advantage of this technology independently for their own benefit. For example, some researchers have outlined the benefits of BIM to the different fields of the AEC industry specifically such us design authoring [3], 3D coordination of different fields [4], dynamic cost estimation [5], building system analysis [6], 4D planning and site utilization analysis [7,8], sustainability analysis [9], digital fabrication [10], and construction safety analysis and management [11], etc. Later on, researchers started to explore the potential value of BIM as an information integration tool between different processes and stakeholders, hence many organizations such as AIA, AGC, and CIC have developed guidelines to facilitate and standardize BIM adoption. [12,13,14]. These studies can be considered as the generic BIM implementation in the AEC industry, but still with the limitation of presenting an effective information model to fulfill the requirements for a smooth information transition and integration between different phases of a construction project. 
This research proposes a new model, the Information Integration Sphere (IIS), to serve as a tool for generating, manipulating and visualizing the information flows of different processes within the BIM modeling. The IIS can comprehensibly illustrate how the different BIM modeling processes interact between each other, identify the data requirements regarding different stakeholders and their respective responsibilities in providing and receiving data, and develop the practical guide for building specificpurpose BIM models for construction projects.

The following sections will first depict the concept and the components of IIS, and then show the steps of building an IIS model. Furthermore, an example will be used to demonstrate how the IIS could be implemented into the construction industry. The conclusion and the future research will finally be presented.

\section{THE INFORMATION INTEGRATION SPHERE}

\section{(IIS)}

The complexity and amount of the information generated during the construction project increase as the project proceeds. Although the BIM model can serve as an information platform that can integrate the information for different stages of the construction project, the stakeholders of the construction project still suffer from spending tremendous amount of time and labor in terms of model development and modification due to the inadequate definition of information requirements. Therefore, there is a need to develop an information model that can flexibly define the information requirements and responsibilities of stakeholders so as to determine the requirements of the model development. The IIS is an information-modeling tool that can generate, manipulate and visualize the flows between different processes. The IIS geometry is similar to that of a sphere, in which the information starts in the center point of the sphere and evolves according to the increasing size of the sphere, as shown in Fig. 1. This feature is identical to the information aggregating process of the construction project. In addition, one of the major contributions of the IIS is the ability to visualize and interpret complex and multidimensional information exchange of a process, in which data flows and data exchanges are not limited only to a linear mode, but instead they could be represented from one dimension to another at different stages of time. The following section will one by one explain the components of the IIS

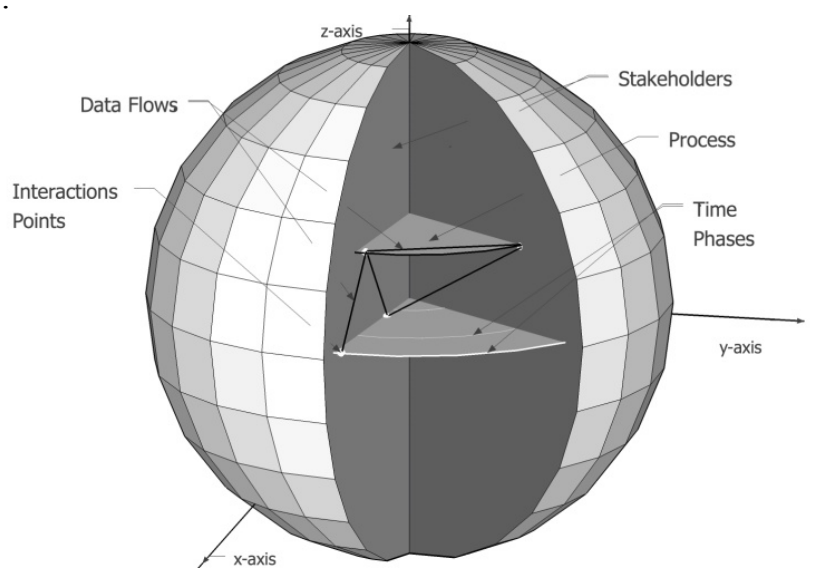

Fig. 1 IIS Components

\subsection{The components}

Timeline, process, stakeholder, and data flows are the four components within the IIS model. The graphic representation of those components within IIS can be found in Fig. 1.

\section{Timeline}

It's the graphic representation showing the passage of time. In the IIS model timeline represents the time where an interaction or exchange of information take place between different stakeholders or processes.

Due to the nature of the geometry of the sphere, the time axis scalability changes according to different processes; meaning that each process has its own timeline, and even though the relationship between different timelines could be established by using the sphere's radius as a reference. Graphically it's represented by circles increasing in diameter located on the $x-y$ plane and starting at the center point of the sphere.

\section{Processes}

Processes are a serious of actions changes or functions bringing about a result. It's a set of transformations of input elements into output elements with specific properties, with the transformations characterized by 
parameters and constraints. Graphically the process in IIS is defined as horizontal planes cutting the sphere parallel to the $\mathrm{x}-\mathrm{y}$ plane, as shown in Fig. 1.

Several stakeholders could participate a process to obtain information transformation or exchange for their own interests. For example, cost estimation is typically the standard process. The project manager could perform cost estimation for the feasibility analysis at the planning stage. For the general contractor, cost estimation could be used to generate dynamic payment reports at the construction stage.

\section{Stakeholder}

Stakeholder could be an individual person or an organization that participates in the information exchange within the construction project. These stakeholders may include the owner, engineers, architect, project manager, and suppliers, etc.

In the IIS stakeholders are defined as intersecting planes parallel to the z-axis, cutting the sphere into slices. These slices contain all the data exchanges regarding each specific stakeholder. Since stakeholders usually play different roles in different process at different stages of the project, the information requirements and responsibilities of the stakeholders could vary within the life of the construction project. Those types of information can be identified by locating the intersection points between the stakeholders and process or timelines.

\section{Data Flow}

Data Flow in the IIS is defined by push-pull knowledge transactions between interaction points. Push mechanisms transfer or input the information into subsequent interaction point while pull mechanisms transfers knowledge to satisfy a request of information from another interaction point.

Data flow in the IIS don't refer to the flow from one process to another but its more about the interactions of stakeholders, processes along a period of time and shows what data are required or must be sent from one interaction point to another in order to interlink and integrate the continuous exchange of information of the whole project life. Data Flow can be classified as:
1. Exchange: when a stakeholder exports or imports reference data or non BIM-related information between Interaction points. For example, exporting the quantity takeoff from a BIM model.

2. Interchange: when a stakeholder exports or imports BIM-related information between interaction points. For example, structural engineer imports an architectural model into structural analysis-BIM software.

Each interaction point between timelines, stakeholders, and processes in the IIS is unique and significant in terms of data exchange and interchange. More interpretations of these intersection points in IIS are defined as the deliverables and explained in the following section.

\subsection{The IIS Deliverables}

The IIS deliverables could be gathered into four major groups: time perspective, stakeholder perspective, process perspective, and data requirements perspective.

\section{Time Perspective}

The time perspective output is an inquiry of the status of different stakeholders' information needs in a certain process at a certain period of time. For example, suppose we want to know the information requirements and responsibilities of different stakeholders at the "planning phase", those details can be found by locating the data flows at the interaction points between various stakeholders and processes at the planning phase". Graphically it is represented as a perimeter along the process in question and passing along all the stakeholders.

\section{Stakeholder Perspective}

This is an inquiry of the how an individual stakeholder interacts with different processes along periods of the project life. Graphically it is represented as a horizontal slice from the intersection of the stakeholder with the sphere. This result can show the requirements and responsibilities of a specific stakeholder for different process at different stages of time. For example, this inquiry should be used to define the responsibilities of one 
stakeholder for providing vital information along a period of time.

\section{Process Perspective}

This inquiry is used for providing information related to a specific process and shows how the interaction of different stakeholders with it. For example this inquiry could be used to show the frequency of usage of a single process, such as cost estimation, along the project life.

\section{Interaction Points Perspective}

This represents the data populated into an interaction point and shows which information is exchanged or interchanged for a specific point. This could be used to generate a complete list of data requirements for the model to ultimately produce "information requirements guide" to be used as a vital planning tool for implementing complex information management systems such as BIM into practice.

\section{IIS STEPS TO BUILD AN IIS}

The steps of building the IIS will be explained, along with an example to illustrate how this model would serve as a tool to build a construction management ready BIM model.

\subsection{Step 1: Identify BIM Objectives and Stakeholders}

First of all, the main objectives for adopting BIM into the traditional workflow should be identified. This step identifies in which areas and phases of the project BIM processes will be applied and which stakeholders will be involved in the exchange of information.

Taking into account the construction management field, some of these objectives may include: increase field productivity, track progress during construction, eliminate field conflicts, compiling an accurate $3 \mathrm{D}$ record model for facility management department, and others.

The key stakeholders regarding BIM information exchanges in the project are: owner, architect, engineers, project manager, general contractor and suppliers.

The different periods of time in which vital information is exchanged are: constructability reviews, bidding and permitting, purchasing and contracting, construction administration, and construction closeout. See Fig.2

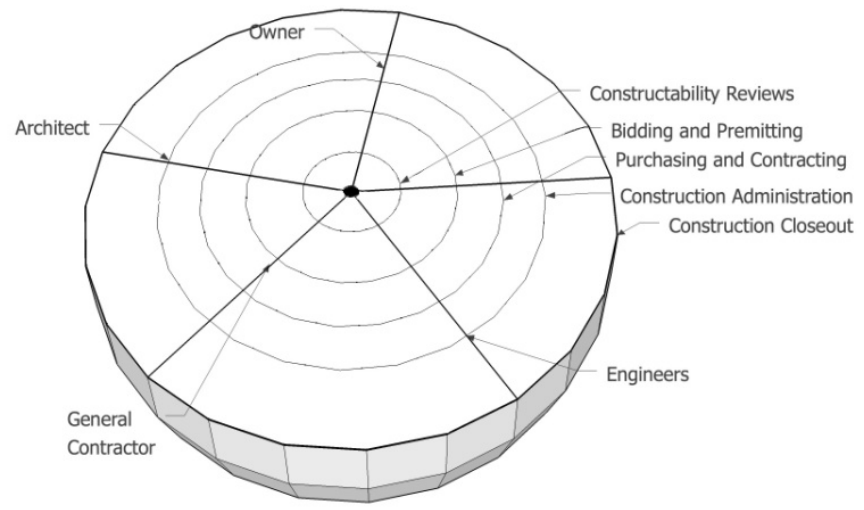

Fig. 2 Timeline and Stakeholders of IIS

\subsection{Step 2: Define BIM Processes.}

In this step, all BIM processes defined earlier will be identified. Activities, controls and information exchanges that will take place in each process, BIM data and nonrelated BIM data inputs and outputs will be listed.

Some of these BIM processes related to the construction management field are: construction system design, phase planning (4D model), cost estimation, 3D coordination, 3D control and planning, code checking, digital fabrication, site utilization planning. See Fig. 3

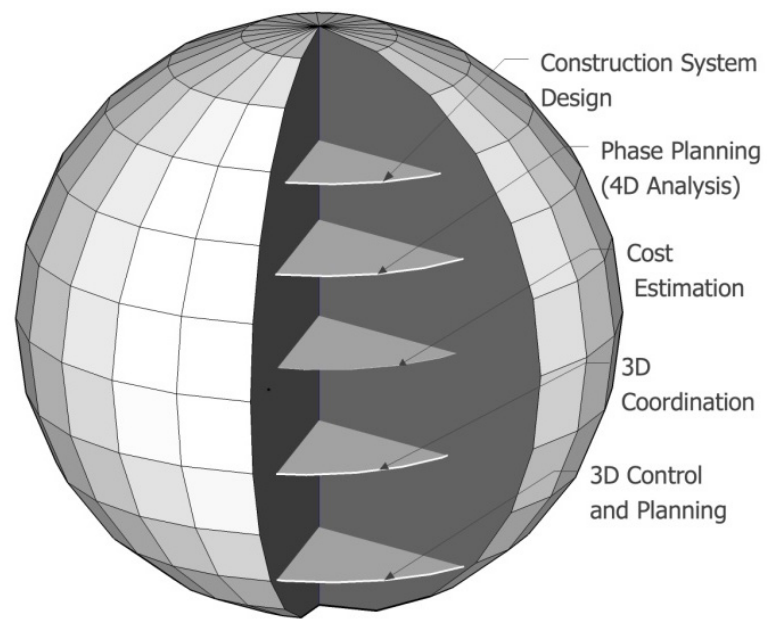

Fig. 3 BIM Processes within the IIS

\subsection{Step 3: Define Information Exchanges.}

In this step, data flows and information exchange should be defined between different stakeholders along the BIM processes, as well as who is responsible for delivering or receiving the data. Any overlaps or data exchange 
inconsistencies between the processes should be identified for more detailed analysis. Fig. 4 shows how data flows from one interaction point to another in the IIS model.

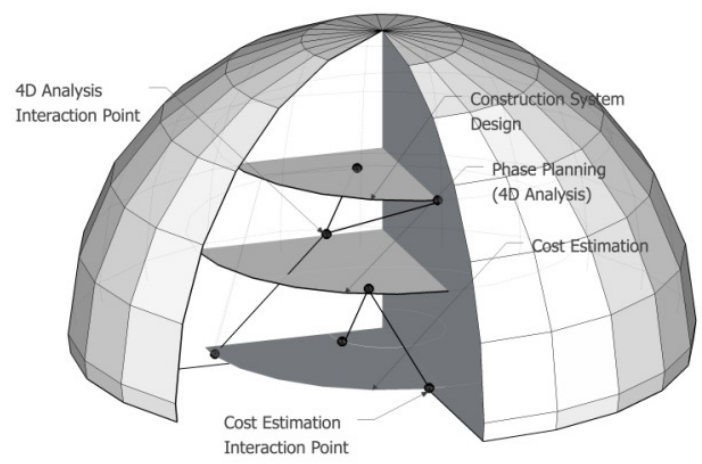

Fig. 4 Data flow in the IIS

\subsection{Step 4: Identify IIS deliverables and products}

Using the different deliverables perspectives explained in section 2.2, the IIS model will be used with the purpose of identifying some of the following information integration outcomes:

1. One of the most important deliverables of the IIS is the information integration products report based on the data flows among the different interaction points. This report helps us identify the data requirements and responsibilities for the different BIM objectives and uses. Fig. 5 shows how the IIS model could be used to identify data flow requirements and define responsibilities when preparing a "4D Analysis" in the "Construction Administration" stage and a "Cost Estimation Report" for the "General Contractor", where the upper part of the table shows the basic information including the name of the integrated product, who is the receiver of the information, phase in which information is needed, and the BIM process applied. The lower part of the table shows who is responsible for providing the information and what kind of attributes or external reference needed to be input in the different components of the building.

2. The stakeholder's data requirements report focuses on one stakeholder in particular; the IIS could identify all data inputs and outputs from one stakeholder involved in different BIM processes. This is highly beneficial in planning the information management for a project because all the information needed from the stakeholders is effectively anticipated.

3. Another result obtained from the IIS model is The Time Data Requirements report. This shows for an instant period of time the different data required from all the stakeholders involved. This report would be best used in the project's information management monitoring and control.

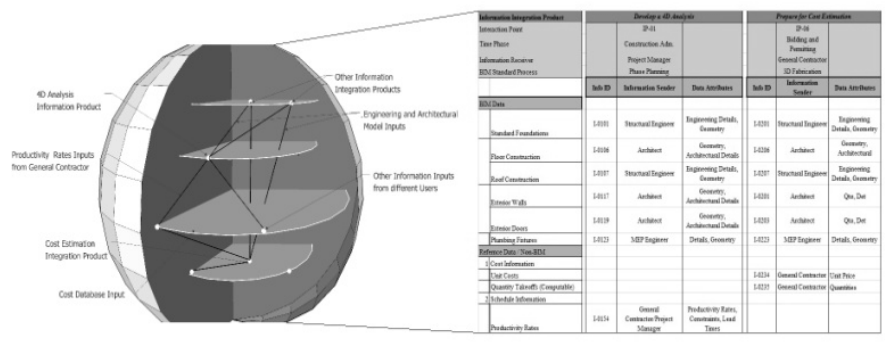

Fig. 5 IIS Information Integration Report

\section{CONCLUSION}

This paper discussed a new approach for implementing BIM into the traditional construction management workflows in order to integrate the various information exchanges between BIM processes and stakeholders. Also the model developed can define the different responsibilities for the stakeholders regarding providing the data in the different phases of the project.

With the implementation of the IIS model, a construction management ready BIM model is achieved where information transition is integrated into an efficient and seamless manner.

Because the IIS was developed with the BIM software, in the future it could be linked to the building's components and used to automatically generate data requirements and responsibilities for the whole lifecycle of the project.

\section{ACKNOWLEDGEMENTS}

This paper supported by National Science Council, Taiwan under the grant NSC-97-2221-E-006-219-MY3.

The authors would also like to thank Taiwan ICDF (International Cooperation and Development Fund) for their unconditional support to this paper.

\section{REFERENCES}

[1] B. Succar, "Building information modeling framework: A research and delivery foundation for industry 
stakeholders", Automation in Construction Vol. 18, pp. 357-375, 2009.

[2] N. Gu, K. London, "Understanding and facilitating BIM adoption in the AEC industry", Automation in Construction Vol. 19 pp. 988-999, 2010.

[3] M. Tardif, "BIM Reaching Forward, Reaching Back". AIArchtiect This Week, Face of the AIA, AIArchitect, 2008. [4] S. Staub - French, A. Khanzode, 3D and 4D Modeling for design and construction coordination: issues and lessons learned", ITcon Vol. 12, pp. 381-407, 2007.

[5] Autodesk Revit, "BIM and Cost Estimating" Press Release, Autodesk, Jan 2007.

[6] Autodesk Revit, "Building Performance Analysis Using Revit”, Autodesk, 2007.

[7] K.W. Chau, M. Anson, J.P. Zhang, "Four-Dimensional Visualization of Construction Scheduling and Site Utilization" Journal of Construction Engineering and Management. Vol. 130 No. 4, pp. 598 - 606, 2004.

[8] D. Heesom, L. Mahdjoubi, "Trends of 4D CAD Applications for Construction Planning" Construction Management and Economics Vol. 22, pp. 171-182, 2004.

[9] S. Azhar, W. A. Carlton, D. Olsen, I. Ahmad, "Building information modeling for sustainable design and LEED rating analysis", Automation in Construction Vol. 20, pp. $217-224,2011$.

[10] R. Rundell. "BIM and Digital Fabrication (1-2-3 Revit Tutorial), 2008.

[11] J.P. Zhang, Z.Z. Hu, "BIM- and 4D-based integrated solution of analysis and management for conflicts and structural safety problems during construction", $\mathrm{Au}$ tomation in Construction, Vol. 20, pp.155-166, 2011.

[12] AIA, Integrated project delivery: a guide, AIA California Council, 2007.

[13] AGC, The Contractors' Guide to BIM - Edition 1, Associated General Contractors of America, (AGC), 2006.

[14] CIC, The Computer Integrated Construction BIM Project Execution Planning Guide - version 2.0, (CIC), 2010. 